\title{
Vernachlässigte Nachfrage aufgezeigt
}

Der zu erwartende Klimawandel wird manche Regionen stark betreffen. Besonders gefährdet ist die Nordseeinsel Sylt. Inwiefern sollte zum Beispiel das Wattenmeer vor den Folgen des Klimawandels geschützt werden? Um die erwarteten Auswirkungen auch ökonomisch zu bewerten, wurde im Rahmen eines interdisziplinären Verbundprojektes im Frühjahr 1999 eine Zahlungsbereitschaftsanalyse durchgeführt, deren Ergebnisse jetzt vorliegen (1).

$\mathrm{V}$

Von Jürgen Meyerboff or allem zwei Schadenskategorien sind für die Bewertung der Auswirkungen des Klimawandels auf die Insel Sylt bedeutend: Erstens Vermögensverluste bei unbebauten Grundstïkken, Gebäuden und öffentlicher Infrastruktur. Sie sind vor allem für die Westseite von Sylt zu erwarten. Zweitens Auswirkungen des Klimawandels auf Natur und Landschaft. Sie sind, wie die Ergebnisse des ökologischen Teilprojektes zeigen, vor allen an der Ostseite zu erwarten. Hier gibt es noch viele Biotope, die für das gesamte Wattenmeer von Bedeutung sind. Sollte der Meeresspiegel steigen und sich dadurch eine stärkere Erosion einstellen, dann wären diese Biotope durch die Wahl „traditioneller“ Küstenschutzmaßnahmen wie der Errichtung und Verstärkung von Deichen bedroht; sie würden in ihrer heutigen Form verloren gehen. Damit stellte sich die Frage, ob der Nutzen aus dem Schutz dieser Biotope die zu erwartenden Kosten für alternative Küstenschutzmaßnahmen übersteigen würde.

Um diesen Nutzen zu ermitteln, wurde eine Zahlungsbereitschaftsanalyse (kontingente Bewertung) durchgeführt. Mit ihr sollten Maßnahmen zum Schutz des Wattenmeeres bewertet werden. Die kontingente Bewertung ist dann das geeignete Bewertungsinstrument, wenn auch nutzungsunabhängige Werte als Bestandteil des gesamten Wertes von Natur und Landschaft ermittelt werden sollen. Diese Werte können einen bedeutenden

\begin{tabular}{|ll|}
\multicolumn{2}{c|}{ Abbildung l: Befragungsdesign } \\
\hline Grundgesamtheit: & Bevölkerung der Bundesrepublik \\
\hline & über 18 Jahre \\
\hline Interviewart: & pers. Interview (25-30 Min.) \\
\hline Informationsvermittlung: & Texte, Karten, Schautafel \\
\hline Zahlungsinstrument: & zunächst nicht vorgegeben \\
\hline Zahlungsmodus: & DM pro Person und Monat \\
Frageform: & Zahlkarte (0,50 - über 300 DM) \\
& Quelle: eigene Darstellung \\
\hline
\end{tabular}

Teil des ökonomischen Wertes von Natur und Landschaft ausmachen. Jedoch ist sie als Bewertungsmethode auch innerhalb der Umwelt- und der ökologischen ökonomie umstritten (2).

\section{- Design der Umfrage}

Da das Wattenmeer eine bedeutende Naturlandschaft in Deutschland ist, wurde die gesamte Bevölkerung in Deutschland als potenziell betroffen von möglichen Veränderungen angesehen. Als nutzungsunabhängiger Wert wurde die Zahlungsbereitschaft von Nicht-Nutzern definiert, also von Personen, die bis zum Zeitpunkt des Interviews die Nordseeküste selber noch nicht besucht hatten. Die Auswahl der 1412 interviewten Haushalte erfolgte über eine dreistufige Zufallsauswahl (3). Abbildung 1 stellt wichtige Elemente des Befragungsdesigns näher dar.

Von der neoklassischen Ökonomik ausgehend ist ein bestimmter Einfluss von Merkmalen der Befragten auf die Zahlungsbereitschaft zu erwarten. So dürfte sie mit der Höhe des Einkommens steigen, und mit höherem Lebensalter oder einer größeren Anzahl von Haushaltsmitgliedern sinken (4). Auch landschaftsbezogene Merkmale wie bisheriger Aufenthalt oder eine geplante Reise dürften positiv wirken. Doch zeigen Untersuchungen, dass allein über diese Faktoren oft nur eine geringe Varianz der Zahlungsbereitschaft erklärt werden kann; zusätzliche Erklärungsfaktoren scheinen erforderlich (5). Daher wurden zusätzlich Einstellungen der Befragten gegenüber Natur, Klimawandel und der Bewertung von Natur mit Geld erhoben. Abbildung 2 auf S. 32 zeigt die Merkmale der interviewten Personen/Haushalte, die in die statistische Auswertung aufgenommen wurden.

\section{Befragungsergebnisse}

Insgesamt hatten 695 Personen bis zum Zeitpunkt des Interviews mindestens einmal die Nordseekü- ste oder eine der vorgelagerten Inseln besucht. Umgekehrt hatten 717 Personen die betreffende Region noch nicht besucht und gelten damit als Nicht-Nutzer. Ihr Anteil nahm mit steigender Entfernung erwartungsgemäß zu. Auf die Frage nach der grundsätzlichen Zahlungsbereitschaft antworteten 336 Personen (23,8 Prozent), dass sie sich grundsätzlich an der Finanzierung eines Schutzprogramms beteiligen würden. Zwei Drittel davon waren Nutzer. Die monatliche Zahlungsbereitschaft lag im Durchschnitt bei 3,50 Mark pro Haushalt. Der Einfluss der unabhängigen Faktoren auf die Höhe der Zahlungsbereitschaft wurde u.a. anhand einer multiplen Regression (OLS) untersucht (6).

Danach haben folgende Faktoren einen signifikant positiven Einfluss auf die Höhe der Zahlungsbereitschaft: das Einkommen, bei den Nutzern die landschaftsbezogenen Einflussgrößen vorherige Aufenthalte in der Region, gute Kenntnisse über die Naturlandschaft Wattenmeer, Wissen über mögliche Folgen des Klimawandels für das Wattenmeer sowie die Teilnahme an einer Wattwanderung. Dagegen zeigen die Faktoren Alter, Haushaltsgröße und auch Entfernung des Wohnortes zum Wattenmeer keinen signifikanten Einfluss. Jedoch weisen die Variablen Alter und Haushaltsgröße das erwartete negative Vorzeichen auf. Die Einstellungen gegenüber der Natur und gegenüber der monetären Bewertung zeigen einen positiven Einfluss. Je stärker die Einstellung von Personen gegenüber der Natur ist und je eher eine Person bereit ist, Geld als einen Bewertungsmaßstab für Natur zu akzeptieren, desto höher fällt die Zahlungsbereitschaft aus. Die Einstellungen zum Klimawandel zeigen dagegen keinen signifikanten Einfluss. Dies könnte darauf hindeuten, dass die Befragten den Schutz des Wattenmeeres weniger mit den möglichen Folgen des Klimawandels in Verbindung bringen als mit dem Wunsch, diese Naturlandschaft generell zu schützen.

Wie sind die Ergebnisse der kontingenten Bewertung einzuschätzen? Die Regressionsanalyse zeigt, dass die Zahlungsbereitschaften nicht rein zufällig sind. Das korrigierte Bestimmtheitsmaß von 0,29 stellt einen relativ guten Wert dar. Deutlich wird aber auch, dass umgekehrt zwei Drittel der Variation nicht erklärt werden. Dieses verbleibende „Rauschen“ ist ein Charakteristikum fast aller kontingenten Bewertungen. Es scheint fraglich, ob deutlich höhere Werte erreichbar sind. Die individuelle Entscheidung über die Zahlungsbereitschaft hängt von einer Vielzahl von Einflüssen 
ab, die im Rahmen der vorgestellten Bewertungsmethode - und wahrscheinlich auch von anderen Methoden - kaum zu erfassen sind. Deutlich wird auch, dass mehr Faktoren die Höhe der Zahlungsbereitschaft bestimmen, als von der ökonomischen Theorie angenommen werden.

\section{Hochrechnung der Zahlungsbereitschaft}

Der einfachste Weg zur Hochrechnung der Ergebnisse ist, die durchschnittliche Zahlungsbereitschaft pro Haushalt mit der Anzahl aller Haushalte in der Bundesrepublik zu multiplizieren. Dies ergibt bei 37,36 Millionen Haushalten (Stand April 1998) und einer mittleren Zahlungsbereitschaft von 3,50 Mark pro Monat, also 42 Mark pro Jahr, insgesamt eine jährliche Zahlungsbereitschaft von 1,55 Milliarden Mark.

Eine konservative Hochrechnung bezieht nur Beträge von Befragten mit ein, die sich sehr sicher oder sicher waren, dass sie den von ihnen genannten Beitrag auch tatsächlich zahlen würden, wenn das Programm umgesetzt wird, und die ihre Zahlung tatsächlich konkret auf das Wattenmeer - und nicht auf allgemeinen Naturschutz - beziehen wollen. In diesem Fall ergibt sich eine aggregierte jährliche Zahlungsbereitschaft von rund 750 Millionen Mark.

Für die gefährdeten Biotope auf der Ostseite von Sylt zeigt sich damit, dass die Kosten für alternative Küstenschutzmassnahmen um ein Vielfaches geringer sind als die Nutzen, die daraus resultieren würden. Die Kosten für Sandvorspülungen, wie sie vom ökologischen Teilprojekt der Fallstu- die vorgeschlagen wurden, belaufen sich auf rund 1,5 Millionen Mark pro Jahr. Dem stehen Nutzen von rund 50 Millionen Mark pro Jahr gegenüber, die aus der konservativen Hochrechnung für das Wattenmeer insgesamt als Wertschätzung für die Flächen auf Sylt abgeleitet wurden.

\section{Fazit}

Die Ergebnisse zeigen, dass eine deutliche Nachfrage nach der Erhaltung von Natur und Landschaft besteht und die Kosten unterlassenen Schutzes sehr hoch sein können. Indem sie dies aufzeigt, leistet die ökonomische Bewertung einen wichtigen Beitrag zur Erhaltung und nachhaltigen Nutzung der Biosphäre, wie dies der Wissenschaftliche Beirat der Bundesregierung für Globale Umweltveränderungen in seinem Jahresgutachten 1999 deutlich macht (7). Fraglich ist aber, ob die so gewonnen Informationen politikkompatibel sind: Diese Nachfrage zu berücksichtigen bedeutet oft, in lokalen Konflikten wie Rückverlegungen von Deichen an der Küste den Interessen der anonymen Nachfrager höhere Priorität einzuräumen als denjenigen vor Ort, die aus ihrer Sicht von den Schutzmaßnahmen negativ betroffen sind.

\section{Anmerkungen}

(1) Die Ergebnisse gehen auf das Forschungsprojekt "Kosten der Klimaänderung auf Sylt" (BMBF-Förderkennzeichen 01 LK 9517/9) im Rahmen des Verbundprojekts „Fallstudie Sylt" des Bund-Länder-Programms „Klimawandel und Küste" zurück. Eine kurze Darstellung findet sich in Hartie, V./ Meyer, I./ Meyerhoff, J.: Kosten einer möglichen Klimaveränderung auf Sylt. In: Daschkeit, A./ Schottes, P. (Hrsg.): Sylt - Klimafolgen für Mensch und Küste, Berlin

\section{Abbildung 2: Konzeptionelles Modell für die Auswertung}

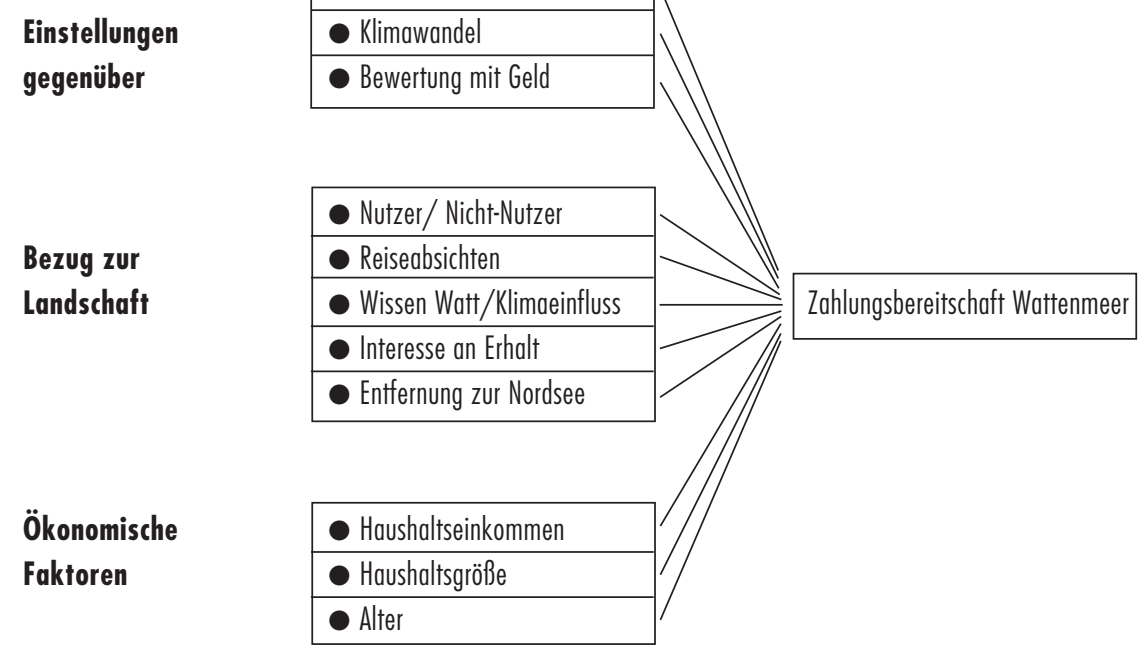

Quelle: in Anlehnung an Wierstra, E.: On the domain of contingent valuation. Enschede, Twente University Press 1996

(im Erscheinen); die längere im Endbericht Hartie, V./ Meyerhoff, J./ Meyer, I.: Kosten einer möglichen Klimaveränderung auf Sylt. Verlag für Wissenschaft und Forschung, Berlin (im Erscheinen).

(2) Vgl. hierzu z.B. Hartje/ Meyerhoff/ Meyer a.a.0.; Biornstad, D.J./ Kahn, J.R. (Hrsg.): The Contingent Valuation of Environmental Resources. Methodological Issues and Research Needs, Cheltenham 1996; sowie Bateman, I.J./ Willis, K.G. (Hrsg.): Contingent Valuation of Environmental Preferences, Oxford 1999. Der Stand der Diskussion zur Anwendung der kontingenten Bewertung im deutschsprachigen Raum ist dargestellt in Elsasser, P./ Meyerhoff, J. (Hrsg.): Kontingente Bewertung - Anwendungen und Erfahrungen im deutschsprachigen Raum, Metropolis, Marburg (im Erscheinen).

(3) Die Stufen waren: 1. Verteilung der Ortsgrößenklassen der Bundesrepublik, 2. Random-Walk zur Auswahl der Haushalte, 3. Schwedenschlüssel zur Auswahl der Zielperson im Haushalt. Erreicht wurde bei rund 2.400 angesprochenen Haushalten eine Ausschöpfungsquote von rund 60 Prozent. (4) Vgl. Carson, R.T./ Flores, N.E./ Meade, N.F.: Contingent valuation: controversies and evidence. In: Environmental and Resource Economics, Vol. 19 (2001), S. 173-210; Spash, C.L.: Environmental Values and Wetland Ecosystems: CVM, Ethics and Attitudes. In: O'Connor, M./ Sang, J.T.K. (Hrsg.): The Valse Project: Full Final Report to the DG-XII, European Commission, Paris 1998.

(5) So z.B. Green, C./ Tunstall, S.: A Psychological Perspective. In: Bateman, I.J./ Willis, K.G. (Hrsg.): Contingent Valuation of Environmental Preferences, Oxford 1999, S. 207-258.

(6) Sogenannte Protestantworten wurden außer für die Hochrechnung der Zahlungsbereitschaften aus der Analyse ausgeschlossen. Danach verblieben 623 Interviews in der Auswertung. Eine Erklärung für den hohen Anteil an Protestantworten dürfte in der zum 1. April 1999 eingeführten Öko-Stever zu finden sein.

(7) Vgl. Wissenschaftlicher Beirat der Bundesregierung Globale Umweltveränderungen: Welt im Wandel: Erhaltung und nachhaltige Nutzung der Biosphäre. Jahresgutachten 1999. Berlin u.a. 2000.

\section{Der Autor}

Jürgen Meyerhoff ist wissenschaftlicher Mitarbeiter am Institut für Management in der Umweltplanung der TU Berlin (IMUP).

Kontakt: IMUP, TU Berlin, Franklinstr. 28/29, 10587 Berlin, Tel. 030/ 314-73322, Fax -73517, E-mail: meyerhoff@imup.tu-berlin.de 
(c) 20I0 Authors; licensee IÖW and oekom verlag. This is an article distributed under the terms of the Creative Commons Attribution Non-Commercial No Derivates License (http://creativecommons.org/licenses/by-nc-nd/3.o/), which permits unrestricted use, distribution, and reproduction in any medium, provided the original work is properly cited. 